\title{
Gliomatosis cerebri or benign intracranial hypertension?
}

\author{
P Weston, J Lear
}

\begin{abstract}
Summary
Benign intracranial hypertension is a condition of obscure aetiology which presents with episodic headaches and nausea. It is a diagnosis based on the exclusion of other intracranial pathology and computed tomography is usually normal. We present a case of gliomatosis cerebri which was initially diagnosed and treated as benign intracranial hypertension.
\end{abstract}

Keywords: benign intracranial hypertension, gliomatosis cerebri, magnetic resonance imaging

\section{Case report}

A 44-year-old woman presented in October 1992 with a two-week history of blurred vision. On examination she was found to be overweight and have a blood pressure of 130/ $90 \mathrm{mmHg}$. Visual acuity was $6 / 18$ in both eyes; visual fields were normal on formal testing. Fundoscopy showed her to have bilateral papilloedema. Her past medical history was unremarkable except for mild diet-controlled diabetes diagnosed two years previously. She was on no regular medication and had never taken the oral contraceptive pill.

Investigations at presentation revealed a normal full blood count and plasma viscosity. Blood biochemistry was also normal except for a blood glucose of $10.9 \mathrm{mmol} / 1$. Her electrocardiogram (ECG) and chest X-ray were both normal. Computed tomography (CT) head scan was reported as normal. Lumbar puncture was performed and showed an opening pressure of $38 \mathrm{~cm}$ cerebrospinal fluid (CSF). There were no cells in the CSF and the protein content was $<0.1 \mathrm{~g} / \mathrm{dl}$. A diagnosis of benign intracranial hypertension was made and she was commenced on a weight-reducing diet; acetazolamide and frusemide were started and she underwent repeated lumbar punctures with the CSF pressure halved at each occasion.

Despite intensive dieting and losing $14 \mathrm{lbs}$ in weight over the next two months her papilloedema did not resolve. Visual acuity worsened to $6 / 36$. The CSF pressure remained elevated at over $35 \mathrm{~cm}$.

In view of the deteriorating visual acuity she

Leicester Royal

Infirmary, Leicester, LE1 5WW, UK

$P$ Weston

J Lear

Accepted 28 December 1994

was referred to the neurosurgeons for insertion of a thecoperitoneal shunt. This was performed in December 1992 without complications. Over the next month there was no improvement in her symptoms or physical signs and in
April 1993 she was readmitted with a severe occipital headache and reduced conscious level. A magnetic resonance imaging (MRI) scan was performed and showed evidence of an infiltrative glioma involving the cerebral hemispheres and brain stem. She underwent a stereotactic brain biopsy that confirmed the diagnosis of gliomatosis cerebri.

Shortly after her biopsy she deteriorated and died. Post-mortem examination revealed a diffusely swollen brain as a result of diffuse infiltration by a malignant glioma.

\section{Discussion}

Gliomatosis cerebri is an uncommon condition in which there is a diffuse overgrowth of neoplastic glial cells with preservation of the underlying cytoarchitecture. ${ }^{1}$ Aetiology is uncertain: a congenital predisposition is suggested by an association with Von Recklinghausen's disease (type 1); varying degrees of differentiation of glial cells of all age groups suggests de novo neoplastic change. ${ }^{1}$ Sex distribution is equal and it is commonest in the fifth decade of life. ${ }^{2}$ It presents with a variable clinical picture but headache and changes of character are often seen. ${ }^{3}$ Diagnosis is difficult as our case illustrates. CT scanning is usually unhelpful, making MRI the diagnostic investigation of choice. Definite ante-mortem diagnosis can be made on brain biopsy but diagnosis is often made only at post-mortem. ${ }^{2}$ Prognosis is poor with a mean survival of 26 months from diagnosis. ${ }^{1}$ The benefit of radiotherapy is difficult to predict, especially in low grade tumours, and chemotherapy is of no benefit although palliative steroids may help.

Benign intracranial hypertension is characterised by raised intracranial pressure with normal CSF in the absence of an intracranial mass. ${ }^{4}$ The prevalence has been estimated as between one and 19 cases per 100000 popula-

\section{Gliomatosis cerebri}

- a rare disease with equal sex distribution

- the commonest symptom is headache and as the disease progresses reduced consciousness, character changes and impaired cognitive function
- aetiology unknown; possible congenital predisposition (some cases associated with Von Reklinghausen's disease) 
tions and women are affected eight times more frequently than men. ${ }^{6}$ There are many associations such as obesity, metabolic and endocrine disorders, drugs and inflammatory disorders. Presentation is usually with headache and nausea; $35 \%$ of patients report visual changes such as horizontal diplopia or tangential visual obscuration. Papilloedema is the most frequent physical finding. ${ }^{7}$ CT scanning is usually normal. ${ }^{8}$ A lumbar puncture shows marked elevation of CSF opening pressure but the fluid constituents are normal.

The aim of treatment is preservation of visual function and correction of any associated conditions such as obesity and endocrine disorders. Medical treatment includes diuretics such as acetazolamide or frusemide but the mainstay of treatment is repeated lumbar punctures to lower the CSF pressure by $50 \%$ each time. Thecoperitoneal shunting results in a resolution of the symptoms and signs within one month. ${ }^{9}$ Fifty per cent of patients, however, require shunt revision in the long term so this treatment is usually reserved for patients with unremitting symptoms or rapid visual loss. ${ }^{8}$

Our patient did not respond to repeated lumbar punctures or to thecoperitoneal shunting. In such a case where the usual natural history of the condition is not followed, we would suggest that, before labeling a patient as having benign intracranial hypertension, an MRI scan of the brain should be performed.

1 Ross IB, Robitaille Y, Villemure J, Tampieri D. Diagnosis and management of gliomatosis cerebri: recent trends. Surg Neurol 1991; 36: 431-40.

2 Kandler RH, Smith CML, Broome JC, Davies-Jones G. Gliomatosis cerebri: a clinical, radiological and pathological Gliomatosis cerebri: a clinical, radiological and patholog
report of four cases. $\operatorname{Br} \mathcal{\text { Neurosurg }}$ 1991; 5: 187-93.

3 report of four cases. Br $\mathcal{f}$ Neurosurg 1991; 5: 187-93. Mineura K, Sasajima T, Kowada M, Shishido F. Innovative approach in the diagnosis of gliomatosis cerebri using carbon-11-L-methionine positron.
Nuclear Med 1991; 32: 726-8.

4 Round R, Keane JR. The minor symptoms of increased intracranial pressure. 101 patients with benign intracranial hypertension. Neurology 1988; 38: 1461-3.

\section{Benign intracranial hypertension}

- a rare disease with a marked female preponderance

- commonest symptom is headache but consciousness is preserved

- papilloedema is a universal finding

- visual field testing may show enlargement of the blind spot

- $30 \%$ of patients develop diplopia most commonly due to VI nerve palsy

- patients are characteristically overweight

- Aetiology

dural sinus thrombosis

pregnancy

drugs (including corticosteroids, tetracycline and nitrofurantoin) empty sella syndrome

the majority of cases have no identifiable cause

\section{Learning points}

- in cases of suspected benign intracranial hypertension which do not respond to conventional treatments the diagnosis should be reconsidered.

- all cases of suspected benign intracranial hypertension should have MRI

5 Durcan FJ, Corbett JJ, Wall M. The incidence of pseudotumour cerebri. Population studies in Iowa and Louisiana. Arch Neurol 1988; 45: 875-7.

6 Digre KB, Corbett JJ. Pseudotumour cerebri in men. Arch Neurol 1988; 45: 866-72.

7 Cody C, Benign intracranial hypertension. Am Family Physician 1992; 45: 1671-8.

8 Susman JL, Benign intracranial hypertension. $f$ Family pract 1990; 30: 290-2.

9 Johnston I, Besser M, Morgan MK, Cerebrospinal fluid diversion in the treatment of benign intracranial hypertension. I Neurosurg 1988; 69: 195-202. 